\title{
STOCHASTIC ORDERS AND MAJORIZATION OF MEAN ORDER STATISTICS
}

\author{
JESÚS DE LA CAL, ${ }^{*}$ Universidad del País Vasco \\ JAVIER CÁRCAMO, ${ }^{* *}$ Universidad Autónoma de Madrid
}

\begin{abstract}
We characterize the (continuous) majorization of integrable functions introduced by Hardy, Littlewood, and Pólya in terms of the (discrete) majorization of finite-dimensional vectors, introduced by the same authors. The most interesting version of this result is the characterization of the (increasing) convex order for integrable random variables in terms of majorization of vectors of expected order statistics. Such a result includes, as particular cases, previous results by Barlow and Proschan and by Alzaid and Proschan, and, in a sense, completes the picture of known results on order statistics. Applications to other stochastic orders are also briefly considered.
\end{abstract}

Keywords: Order statistic; stochastic ordering; convex order; increasing convex order; discrete majorization; continuous majorization; Schur-convex function

2000 Mathematics Subject Classification: Primary 60E15; 60E99; 62E99; 26D1

\section{Introduction: majorization}

Denote by $L^{1}((0,1))$ the set of all real Lebesgue-integrable functions on $(0,1)$, and let $\mathcal{L}_{\text {cx }}(I)$ be the set of all convex functions on the interval $I$. If $f, g \in L^{1}((0,1))$ take values in the interval $I$, we say that $f$ is majorized by $g$, written $f \prec g$, if the inequality

$$
\int_{0}^{1} \phi(f(t)) \mathrm{d} t \leq \int_{0}^{1} \phi(g(t)) \mathrm{d} t
$$

holds for all $\phi \in \mathcal{L}_{\mathrm{cx}}(I)$ for which the integrals exist. The relation ' $\prec$ ', called (continuous) majorization, was introduced by Hardy et al. [7], [8], [9]. It is a preorder, since we have $f \prec g$ and $g \prec f$ whenever $f^{*}=g^{*}$ almost everywhere, where $f^{*}$ and $g^{*}$ are the increasing rearrangements of $f$ and $g$, respectively. We recall that

$$
f^{*}(t):=\inf \{x: \mathrm{P}(f(U) \leq x) \geq t\}, \quad 0<t<1,
$$

where $U$ is a random variable uniform on $(0,1)$, and we have

$$
\int_{0}^{1} f(t) \mathrm{d} t=\int_{0}^{1} f^{*}(t) \mathrm{d} t
$$

moreover, $f^{*}=f$ almost everywhere if and only if $f$ is increasing (which term is used synonymously with 'nondecreasing' throughout the paper). The weak (continuous) majorization ' $\prec_{\mathrm{w}}$ '

Received 16 November 2005; revision received 17 March 2006.

* Postal address: Departamento de Matemática Aplicada y Estadística e Investigación Operativa, Facultad de Ciencia y Tecnología, Universidad del País Vasco, Apartado 644, 48080 Bilbao, Spain. Email address: jesus.delacal@ehu.es

** Postal address: Departamento de Matemáticas, Facultad de Ciencias, Universidad Autónoma de Madrid, 28049 Madrid, Spain. Email address: javier.carcamo@uam.es 
is defined in the same way as ' $\prec$ ', with $\mathcal{L}_{\text {cx }}(I)$ replaced by $\mathcal{L}_{\text {icx }}(I)$, the set of all increasing functions in $\mathcal{L}_{\mathrm{cx}}(I)$. It is also well known [5] that the condition $f \prec g$ is equivalent to

$$
\int_{x}^{1}\left(f^{*}(t)-g^{*}(t)\right) \mathrm{d} t \leq 0, \quad 0 \leq x \leq 1, \quad \text { with equality for } x=0 .
$$

The characterization for $f \prec_{\mathrm{w}} g$ is the same, without the restriction for $x=0$.

Hardy et al. [7] also introduced the (discrete) majorization for finite-dimensional vectors defined as follows: for $\boldsymbol{x}, \boldsymbol{y} \in \mathbb{R}^{n}$, we write $\boldsymbol{x} \prec \boldsymbol{y}$ if

$$
S_{k: n}(\boldsymbol{x}) \leq S_{k: n}(\boldsymbol{y}), \quad 1 \leq k \leq n, \quad \text { with equality for } k=n,
$$

where

$$
S_{k: n}(\boldsymbol{x}):=\sum_{i=n+1-k}^{n} x_{i: n}, \quad \boldsymbol{x} \in \mathbb{R}^{n},
$$

and $x_{1: n} \leq x_{2: n} \leq \cdots \leq x_{n: n}$ are the components of $\boldsymbol{x}$ arranged in increasing order. Again, this relation is a preorder because $\boldsymbol{x} \prec \boldsymbol{y}$ and $\boldsymbol{y} \prec \boldsymbol{x}$ only imply that $\boldsymbol{y}$ can be obtained from $\boldsymbol{x}$ by a permutation of the components; however, it is a partial order on the set of all $\boldsymbol{x}:=\left(x_{1}, \ldots, x_{n}\right) \in \mathbb{R}^{n}$ such that $x_{1} \leq x_{2} \leq \cdots \leq x_{n}$. The weak majorization $\boldsymbol{x} \prec_{\mathrm{w}} \boldsymbol{y}$ is defined by (1.3) without the restriction for $k=n$.

Remark 1.1. For a comprehensive treatment of the preceding notions and their many developments we refer the reader to the classic book by Marshall and Olkin [12]. In particular, it can be seen there that, for $\boldsymbol{x}, \boldsymbol{y} \in I^{n}$, each of the following assertions is equivalent to saying that $\boldsymbol{x} \prec \boldsymbol{y}$ :

(a) $\phi(x) \leq \phi(y)$ for each Schur-convex function $\phi$ on $I^{n}$;

(b) $\phi(\boldsymbol{x}) \leq \phi(\boldsymbol{y})$ for each symmetric quasiconvex function $\phi$ on $I^{n}$;

(c) $\sum_{i=1}^{n} g\left(x_{i}\right) \leq \sum_{i=1}^{n} g\left(y_{i}\right)$ for each convex function $g$ on $I$.

The equivalence remains true if ' $\prec$ ' is replaced by ' $\prec_{\mathrm{w}}$ ' and the functions in (a)-(c) are assumed to be increasing. Observe that condition (c) is the discrete analogue of (1.1).

It is easy to see that discrete majorization can be characterized in terms of continuous majorization (see Remark 2.3, below). Conversely, in this paper we provide a characterization of continuous majorization in terms of discrete majorization. In order to give a precise statement of our result, we introduce some notation. For $s$ and $r, s+1>r>0$, we denote by $\beta_{r: s}$ a random variable having the $\operatorname{Beta}(r, s+1-r)$ density given by

$$
b_{r: s}(x):=\frac{\Gamma(s+1)}{\Gamma(r) \Gamma(s+1-r)} x^{r-1}(1-x)^{s-r} 1_{(0,1)}(x),
$$

where $1_{A}$ stands for the indicator function of the set $A$; when $s+1>0, \beta_{0: s}$ represents the random variable degenerate at 0 . Now, for $f \in L^{1}((0,1))$ we set

$$
f_{n}:=\left(f_{1: n}, \ldots, f_{n: n}\right), \quad n=1,2, \ldots
$$

where

$$
f_{i: n}:=\mathrm{E} f^{*}\left(\beta_{i: n}\right), \quad 1 \leq i \leq n .
$$

The characterization is stated as follows. 
Theorem 1.1. For $f, g \in L^{1}((0,1))$, the following three assertions are mutually equivalent:

(a) $f \prec g$;

(b) $\boldsymbol{f}_{n} \prec \boldsymbol{g}_{n}$ for all $n \geq 1$;

(c) $\boldsymbol{f}_{n} \prec \boldsymbol{g}_{n}$ for infinitely many values of $n$.

Moreover, the equivalence remains true if ' $\prec$ ' is replaced by ' $\prec_{\mathrm{w}}$ '.

The proof of this result is deferred to the last section of the paper. In the next section we deal with the most interesting version of Theorem 1.1, namely the characterization of the (increasing) convex order for integrable random variables in terms of expected order statistics. It contains previous results that can be found in the literature and, in a sense, completes the picture of known results on order statistics. Section 3 collects related remarks concerning other stochastic orders.

\section{Convex orderings and expected order statistics}

Let $X$ and $Y$ be integrable $I$-valued random variables having distribution functions $F$ and $G$, respectively, and denote by $F^{-1}$ and $G^{-1}$ the respective quantile functions, i.e.

$$
F^{-1}(t):=\inf \{x: F(x) \geq t\}, \quad 0<t<1 .
$$

It is said that $X$ is smaller than $Y$ in the convex order, written $X \leq_{\mathrm{cx}} Y$, if $\mathrm{E} \phi(X) \leq \mathrm{E} \phi(Y)$ for all $\phi \in \mathcal{L}_{\mathrm{cx}}(I)$ for which the expectations exist. When $\mathcal{L}_{\mathrm{cx}}(I)$ is replaced by $\mathcal{L}_{\text {icx }}(I)$, we have the definition of increasing convex order, denoted by ' $\leq_{\text {icx }}$ '. These stochastic orders are well known in the literature, sometimes under other names; for instance, in the theory of decision under risk, ' $\leq_{\mathrm{icx}}$ ' and ' $\leq_{\mathrm{cx}}$ ' are respectively called stop-loss order and stop-loss order with equal means. Accounts of their numerous properties and applications to several fields, as well as further references, can be found in [13], [15], and [18]. In particular, $X \leq_{\mathrm{icx}} Y$ implies that $\mathrm{E} X \leq \mathrm{E} Y$, and we have $X \leq_{\mathrm{cx}} Y$ if and only if $X \leq_{\mathrm{icx}} Y$ and $\mathrm{E} X=\mathrm{E} Y$.

If $U$ is a random variable having the uniform distribution on $(0,1)$, then $F^{-1}(U)$ and $G^{-1}(U)$ have the same distributions as $X$ and $Y$, respectively. It follows that

$$
X \leq_{\mathrm{cx}} Y \quad \text { if and only if } \quad F^{-1} \prec G^{-1}
$$

and this assertion remains true if ' $\leq_{\mathrm{cx}}$ ' and ' $\prec$ ' are respectively replaced by ' $\leq_{\mathrm{icx}}$ ' and ' $\prec_{\mathrm{w}}$ '.

This connection between the (increasing) convex order and (weak) continuous majorization is known in the literature (see, for instance, [14]; [16] contains the criteria for ' $\leq_{\mathrm{cx}}$ ' and ' $\leq_{\mathrm{icx}}$ ' based on (1.2) without any mention of majorization).

On the other hand, a quantile function is increasing, so it coincides (almost everywhere) with its corresponding increasing rearrangement. Therefore, by (1.5) and [2, Equation (5.2.8)], we have, for all $n \geq 1$ and $i, 1 \leq i \leq n$,

$$
F_{i: n}^{-1}=\mathrm{E}\left(F^{-1}\left(\beta_{i: n}\right)\right)=\mathrm{E} X_{i: n}, \quad G_{i: n}^{-1}=\mathrm{E}\left(G^{-1}\left(\beta_{i: n}\right)\right)=\mathrm{E} Y_{i: n},
$$

where $X_{i: n}$ and $Y_{i: n}$ are the respective $i$ th order statistics associated with random samples of size $n, \boldsymbol{X}_{n}:=\left(X_{1}, \ldots, X_{n}\right)$ and $\boldsymbol{Y}_{n}:=\left(Y_{1}, \ldots, Y_{n}\right)$, of $X$ and $Y$.

Thus, Theorem 1.1 (combined with Remark 1.1) yields the following characterization of the (increasing) convex order in terms of majorization of vectors of expected order statistics. 
Corollary 2.1. Let $X$ and $Y$ be integrable I-valued random variables and, for $n \geq 1$, set

$$
\boldsymbol{\mu}_{n}^{X}:=\left(\mathrm{E} X_{1: n}, \ldots, \mathrm{E} X_{n: n}\right), \quad \boldsymbol{\mu}_{n}^{Y}:=\left(\mathrm{E} Y_{1: n}, \ldots, \mathrm{E} Y_{n: n}\right)
$$

The following assertions are mutually equivalent:

(a) $X \leq_{\mathrm{cx}} Y$;

(b) $\boldsymbol{\mu}_{n}^{X} \prec \boldsymbol{\mu}_{n}^{Y}$ for all $n \geq 1$;

(c) $\boldsymbol{\mu}_{n}^{X} \prec \boldsymbol{\mu}_{n}^{Y}$ for infinitely many values of $n$;

(d) $\phi\left(\mu_{n}^{X}\right) \leq \phi\left(\mu_{n}^{Y}\right)$ for all $n \geq 1$ and each Schur-convex function $\phi$ on $I^{n}$;

(e) $\phi\left(\mu_{n}^{X}\right) \leq \phi\left(\mu_{n}^{Y}\right)$ for all $n \geq 1$ and each symmetric quasiconvex function $\phi$ on $I^{n}$;

(f) $\sum_{i=1}^{n} g\left(\mathrm{E} X_{i: n}\right) \leq \sum_{i=1}^{n} g\left(\mathrm{E} Y_{i: n}\right)$ for all $n \geq 1$ and each convex function $g$ on $I$.

Moreover, the equivalence remains true if ' $\leq_{\mathrm{cx}}$ ' and ' $\prec$ ' are replaced by ' $\leq_{\mathrm{icx}}$ ' and ' $\prec_{\mathrm{w}}$ ', respectively, and the functions in $(d)-(f)$ are assumed to be increasing.

Remark 2.1. Corollary 2.1 is actually an equivalent version of Theorem 1.1, as it immediately follows from (2.1) and the fact that each left-continuous, increasing real function on $(0,1)$ is the quantile function of a probability distribution function.

Remark 2.2. Although the sequences $\left(\mathrm{E} X_{n: n}\right)_{n}$ and $\left(\mathrm{E} Y_{n: n}\right)_{n}$ characterize the distributions of $X$ and $Y$ (see [10]), expected maxima are not sufficient to determine the comparability in the convex sense of the parent distributions, as the following simple example shows. Let $X$ and $Y$ be uniform, discrete random variables taking the values $-\frac{3}{4}, \frac{3}{4}$ and $-1,0,1$, respectively. Both $X$ and $Y$ have mean 0 , and it is readily checked that

$$
\mathrm{E} X_{n: n}=\frac{3}{4}-\frac{3}{2^{n+1}}<1-\frac{1+2^{n}}{3^{n}}=\mathrm{E} Y_{n: n}
$$

for all $n \geq 2$, but we have $\mathrm{E}|X|=\frac{3}{4}>\frac{2}{3}=\mathrm{E}|Y|$ and, hence, $X$ and $Y$ are not comparable for ' $\leq_{\mathrm{cx}}$ '. Actually, the condition $\mathrm{E} X_{n: n} \leq \mathrm{E} Y_{n: n}, n \geq 1$, defines a different ordering for random variables, which has been discussed in [6] and [19].

Remark 2.3. According to (2.1) and what was said in Remark 1.1, for $\boldsymbol{x}, \boldsymbol{y} \in \mathbb{R}^{n}$ we assert that

$$
\boldsymbol{x} \prec \boldsymbol{y} \quad \text { if and only if } \quad X \leq_{\mathrm{cx}} Y \quad \text { if and only if } \quad F^{-1} \prec G^{-1},
$$

where $X$ and $Y$ are uniform, discrete random variables taking the values $x_{1}, \ldots, x_{n}$ and $y_{1}, \ldots, y_{n}$, respectively, and $F$ and $G$ are the respective distribution functions. In this way, discrete majorization is characterized in terms of continuous majorization.

Corollary 2.1 contains some results already known in the literature. We recall that $X$ is said to be smaller than $Y$ in the dispersive order, written $X \leq_{\operatorname{disp}} Y$, if

$$
F^{-1}(y)-F^{-1}(x) \leq G^{-1}(y)-G^{-1}(x), \quad 0<x<y<1 .
$$

According to [15, Theorem 2.B.10] (under the assumption of integrability), the relation $X \leq_{\text {disp }} Y$ implies that $X-\mathrm{E} X \leq_{\mathrm{cx}} Y-\mathrm{E} Y$. Thus, Corollary 2.1 implies the following result, shown by Alzaid and Proschan [1].

Corollary 2.2. If $\mathrm{E} X=\mathrm{E} Y$ and $X \leq \operatorname{disp} Y$, then $\boldsymbol{\mu}_{n}^{X} \prec \boldsymbol{\mu}_{n}^{Y}$ for all $n \geq 1$. 
On the other hand, when $X$ and $Y$ are nonnegative random variables, $X$ is said to be smaller than $Y$ in the star order, written $X \leq_{*} Y$, if the function $G^{-1} \circ F$ is star shaped, i.e. if $G^{-1}(F(x)) / x$ is increasing in $(0, \infty)$. It is known [15, Theorem 3.C.4] that, if $X$ and $Y$ have positive means, the assumption $X \leq_{*} Y$ implies that $X / \mathrm{E} X \leq_{\mathrm{cx}} Y / \mathrm{E} Y$. Therefore, the following result of [4] is also a direct consequence of Corollary 2.1.

Corollary 2.3. If $\mathrm{E} X=\mathrm{E} Y$ and $X \leq_{*} Y$, then $\boldsymbol{\mu}_{n}^{X} \prec \boldsymbol{\mu}_{n}^{Y}$ for all $n \geq 1$.

Under assumptions slightly stronger than those in the preceding corollary, Szekli [17] has obtained some results on the comparison of higher moments of order statistics. In this direction, we make the following assertion.

Corollary 2.4. Let $X$ and $Y$ be integrable $I$-valued random variables such that $X \leq_{\mathrm{icx}} Y$. Then, for all $g \in \mathcal{L}_{\mathrm{icx}}(I)$ such that $g(X)$ and $g(Y)$ are integrable, we have

$$
\left(\mathrm{E} g\left(X_{1: n}\right), \ldots, \mathrm{E} g\left(X_{n: n}\right)\right) \prec_{\mathrm{w}}\left(\mathrm{E} g\left(Y_{1: n}\right), \ldots, \mathrm{E} g\left(Y_{n: n}\right)\right) \text {. }
$$

Proof. From the assumptions, we have $g(X) \leq_{\text {icx }} g(Y)$, and Corollary 2.1 yields

$$
\boldsymbol{\mu}_{n}^{g(X)} \prec_{\mathrm{w}} \boldsymbol{\mu}_{n}^{g(Y)} .
$$

Finally, it suffices to observe that the $i$ th order statistics of $g(X)$ and $g(Y)$ are just $g\left(X_{i: n}\right)$ and, respectively, $g\left(Y_{i: n}\right)$.

This result applies, in particular, when $g(x):=x^{r}$, if $r$ is an odd positive integer or if $r>1$ and $I \subset[0, \infty)$.

\section{Other stochastic orders}

In this section we make some remarks on other stochastic orders usually considered in the literature which are closely related to the (increasing) convex order. For further information on these, we refer the reader to [13] and [15].

The (increasing) concave order. It is said that $X$ is smaller than $Y$ in concave order, written $X \leq_{\mathrm{cv}} Y$, if $\mathrm{E} \phi(X) \leq \mathrm{E} \phi(Y)$ for each real concave function $\phi$ (on $I$ ) for which both expectations exist. When 'concave' is replaced by 'increasing concave' here, we have the definition of increasing concave order, denoted by ' $\leq$ icv', which is extensively used in economics, where it is called second-order stochastic dominance. Since it is clear that

$$
X \leq_{\mathrm{cv}} Y \text { if and only if } Y \leq_{\mathrm{cx}} X
$$

and

$$
X \leq_{\text {icv }} Y \text { if and only if }-Y \leq_{\text {icx }}-X,
$$

Corollary 2.1 immediately supplies characterizations for the (increasing) concave order in terms of expected order statistics. We omit the obvious details.

The dilation order and the Lorenz order. The convex order is not location free. This means that only random variables having the same mean are comparable in convex order. To overcome this drawback, two alternatives have been proposed in the literature: the dilation order, ' $\leq_{\text {dil }}$ ', and the Lorenz order, ' $\leq_{\text {Lor' }}$. The relation $X \leq_{\text {dil }} Y$ is defined by the condition $X-\mathrm{E} X \leq_{\mathrm{cx}} Y-\mathrm{E} Y$, and the relation $X \leq_{\mathrm{Lor}} Y$ is defined by the condition $X / \mathrm{E} X \leq_{\mathrm{cx}} Y / \mathrm{E} Y$, provided that $X$ and $Y$ are nonnegative random variables with positive means. As in the preceding cases, Corollary 2.1 serves to characterize these stochastic orders in terms of expected order statistics. 
Among other applications, characterizations increase our ability to check a given relationship easily. The following corollary illustrates this point in the topic under consideration.

Corollary 3.1. Let $Z$ be an integrable random variable. Then, for $0 \leq r<s$, we have $r Z \leq$ dil $s Z$.

Proof. For $n \geq 1$, let

$$
\boldsymbol{m}_{n}:=(\mathrm{E} Z, \ldots, \mathrm{E} Z) \in \mathbb{R}^{n}, \quad \boldsymbol{\mu}_{n}:=\left(\mathrm{E} Z_{1: n}, \ldots, \mathrm{E} Z_{n: n}\right) .
$$

Since $\boldsymbol{m}_{n} \prec \boldsymbol{\mu}_{n}$, we have $(s-r) \boldsymbol{m}_{n} \prec(s-r) \boldsymbol{\mu}_{n}$ and, therefore, $r \boldsymbol{\mu}_{n}-r \boldsymbol{m}_{n} \prec s \boldsymbol{\mu}_{n}-s \boldsymbol{m}_{n}$. By Corollary 2.1, this shows that $r Z-\mathrm{E}(r Z) \leq_{\mathrm{cx}} s Z-\mathrm{E}(s Z)$, i.e. $r Z \leq_{\text {dil }} s Z$.

Remark 3.1. In particular, two random variables having gamma (or Weibull) distributions differing only in the scale parameter are always comparable in dilation order. The specific case of exponential distributions was considered in [3, Example 2.5], where it was remarked that 'a direct verification of this fact is not simple'.

\section{Proof of Theorem 1.1}

We start by establishing the necessary auxiliary results. The notation is the same as in (1.5).

Lemma 4.1. If $f \in L^{1}((0,1))$ then

$$
\mathrm{E} f^{*}\left(\beta_{r: s}\right) \leq \mathrm{E} f^{*}\left(\beta_{r^{\prime}: s}\right), \quad s+1>r^{\prime}>r \geq 1 .
$$

In particular, we have $f_{1: n} \leq f_{2: n} \leq \cdots \leq f_{n: n}$.

Proof. From the integrability of $f^{*}$, it is immediate that the expectations in the statement exist and are finite. Now let $\left\{\gamma_{t}: t \geq 0\right\}$ be a standard gamma process, i.e. a stochastic process starting at 0 , having independent stationary increments, and such that, for each $t>0, \gamma_{t}$ has the gamma distribution with density

$$
g_{t}(x):=\frac{x^{t-1} \mathrm{e}^{-x}}{\Gamma(t)} 1_{(0, \infty)}(x) .
$$

Then $\beta_{r: s}$ has the same distribution as $\gamma_{r} / \gamma_{s+1}$. Since

$$
\frac{\gamma_{r}}{\gamma_{s+1}} \leq \frac{\gamma_{r^{\prime}}}{\gamma_{s+1}} \quad \text { almost surely, } \quad s+1>r^{\prime}>r \geq 0,
$$

and $f^{*}$ is increasing, the conclusion follows.

Lemma 4.2. Let $r(\cdot)$ and $s(\cdot)$ be positive functions on $(0, \infty)$ increasing to $\infty$ and such that $s(\cdot)+1>r(\cdot)$. If $\lim _{t \rightarrow \infty} r(t) / s(t)=l \in[0,1]$, then $\beta_{r(t): s(t)}$ converges in distribution to $l$ as $t \rightarrow \infty$.

Proof. Since $\beta_{r: s}$ has the same distribution as $\gamma_{r} / \gamma_{s+1}$, where $\left\{\gamma_{t}: t \geq 0\right\}$ is a standard gamma process, the conclusion follows from the strong law of large numbers, i.e.

$$
\lim _{t \rightarrow \infty} \frac{\gamma_{t}}{t}=1 \quad \text { almost surely. }
$$


Lemma 4.3. If $f \in L^{1}((0,1))$ then, for $n \geq 1$ and $1 \leq k \leq n$,

$$
S_{k: n}\left(f_{n}\right)=n \int_{0}^{1} f^{*}(t) B_{n-k: n-1}(t) \mathrm{d} t,
$$

where $S_{k: n}$ is as given in (1.4) and $B_{n-k: n-1}(\cdot):=\mathrm{P}\left(\beta_{n-k: n-1} \leq \cdot\right)$ is the distribution function of $\beta_{n-k: n-1}$.

Proof. Fix $n$ and $k$. From (1.5) and Lemma 4.1, we have

$$
S_{k: n}\left(f_{n}\right)=\int_{0}^{1} f^{*}(t)\left(\sum_{i=n+1-k}^{n} b_{i: n}(t)\right) \mathrm{d} t .
$$

Now, for $0<t<1$,

$$
\begin{aligned}
\sum_{i=n+1-k}^{n} b_{i: n}(t) & =n \sum_{i=n+1-k}^{n}\left(\begin{array}{c}
n-1 \\
i-1
\end{array}\right) t^{i-1}(1-t)^{n-i} \\
& =n \sum_{i=n-k}^{n-1}\left(\begin{array}{c}
n-1 \\
i
\end{array}\right) t^{i}(1-t)^{n-1-i} \\
& =n \mathrm{P}\left(\beta_{n-k: n-1} \leq t\right)
\end{aligned}
$$

the last equality following from [11, Equation (3.37)]. This completes the proof of the lemma.

Lemma 4.4. Let $f, g \in L^{1}((0,1))$ and assume that

$$
\int_{a / b}^{1}\left(f^{*}(t)-g^{*}(t)\right) \mathrm{d} t \begin{cases}<0 & (\text { case }(i)) \\ >0 & (\text { case }(i i))\end{cases}
$$

where $a$ and $b$ are integer numbers such that $0 \leq a<b$. Then there exists an integer, $m_{0} \geq 1$, such that

$S_{c m+r: b m+r}\left(\boldsymbol{f}_{b m+r}\right)-S_{c m+r: b m+r}\left(\boldsymbol{g}_{b m+r}\right)\left\{\begin{array}{l}<0 \text { incase }(i), \\ >0 \quad \text { incase }(i i),\end{array} \quad m \geq m_{0}, r=0,1, \ldots, b-1\right.$,

where $c:=b-a$. In particular, when $a=0$ we can take $m_{0}=1$.

Proof. By Lemma 4.3, for all $m \geq 1$ and $r=0,1, \ldots, b-1$ we have

$$
\begin{aligned}
(b m+ & r)^{-1}\left(S_{c m+r: b m+r}\left(\boldsymbol{f}_{b m+r}\right)-S_{c m+r: b m+r}\left(\boldsymbol{g}_{b m+r}\right)\right) \\
& =\int_{0}^{1}\left(f^{*}(t)-g^{*}(t)\right) B_{a m: b m+r-1}(t) \mathrm{d} t,
\end{aligned}
$$

where $B_{a m: b m+r-1}$ is the distribution function of the variable $\beta_{a m: b m+r-1}$. For $a=0$, the conclusion directly follows from the hypothesis, because $\beta_{0: b m+r-1}$ is degenerate at 0 . For $a>0$, by Lemma 4.2 we have

$$
\lim _{m \rightarrow \infty} B_{a m: b m+r-1}(t)=1_{[a / b, \infty)}(t), \quad t \neq \frac{a}{b}, r=0,1, \ldots, b-1 .
$$


Since $f^{*}-g^{*}$ is integrable and $B_{a m: b m+r-1}$ is bounded by 1 , by dominated convergence we obtain

$$
\lim _{m \rightarrow \infty} \int_{0}^{1}\left(f^{*}(t)-g^{*}(t)\right) B_{a m: b m+r-1}(t) \mathrm{d} t=\int_{a / b}^{1}\left(f^{*}(t)-g^{*}(t)\right) \mathrm{d} t
$$

for $r=0,1, \ldots, b-1$, and the conclusion also follows in this case.

We are now in a position to give a short proof of the theorem.

Proof of Theorem 1.1. Let $f, g \in L^{1}((0,1))$ and $n \geq 1$. From Lemma 4.3 and Fubini's theorem, we have, for $1 \leq k<n$,

$$
\begin{aligned}
S_{k: n}\left(\boldsymbol{f}_{n}\right)-S_{k: n}\left(\boldsymbol{g}_{n}\right) & =n \int_{0}^{1}\left(f^{*}(t)-g^{*}(t)\right)\left(\int_{0}^{t} b_{n-k: n-1}(u) \mathrm{d} u\right) \mathrm{d} t \\
& =n \int_{0}^{1} b_{n-k: n-1}(u)\left(\int_{u}^{1}\left(f^{*}(t)-g^{*}(t)\right) \mathrm{d} t\right) \mathrm{d} u,
\end{aligned}
$$

and we also have

$$
S_{n: n}\left(\boldsymbol{f}_{n}\right)-S_{n: n}\left(\boldsymbol{g}_{n}\right)=n \int_{0}^{1}\left(f^{*}(t)-g^{*}(t)\right) \mathrm{d} t .
$$

In view of (1.2), we conclude that assertion (a) of Theorem 1.1 implies assertion (b).

Now assume that assertion (a) does not hold. Then, again by (1.2), we have either

$$
\int_{0}^{1}\left(f^{*}(t)-g^{*}(t)\right) \mathrm{d} t<0 \quad \text { or } \quad \int_{r}^{1}\left(f^{*}(t)-g^{*}(t)\right) \mathrm{d} t>0
$$

for some $r \in[0,1)$ which, by continuity, can be assumed to be a rational number. In any case, we conclude from Lemma 4.4 that there exists an integer $n_{0}$ such that $\boldsymbol{f}_{n} \nprec \boldsymbol{g}_{n}$ for all $n \geq n_{0}$; i.e. assertion (c) is false. This shows that (c) implies (a). Since (b) trivially implies (c), the proof of the first part of the theorem is complete. The proof of the second part is just the same with the obvious modifications.

\section{Acknowledgement}

We thank the referee for suggesting improvements on the presentation and readability of the paper and for pointing out reference [17], which inspired Corollary 2.4.

\section{References}

[1] Alzaid, A. A. and Proschan, F. (1992). Dispersivity and stochastic majorization. Statist. Prob. Lett. 13, 275-278.

[2] Arnold, B. C., Balakrishnan, N. and Nagaraja, H. N. (1992). A First Course in Order Statistics. John Wiley, New York.

[3] Belzunce, F., Pellerey, F., Ruiz, J. M. and Shaked, M. (1997). The dilation order, the dispersive order, and orderings of residual lives. Statist. Prob. Lett. 33, 263-275.

[4] Barlow, R. E. And Proschan, F. (1966). Inequalities for linear combinations of order statistics from restricted families. Ann. Math. Statist. 37, 1574-1592.

[5] Chong, K.-M. (1974). Some extensions of a theorem of Hardy, Littlewood and Pólya and their applications. Canad. J. Math. 26, 1321-1340.

[6] Downey, P. J. AND MaIER, R. S. (1992). Orderings arising from expected extremes, with an application. In Stochastic Inequalities (IMS Lecture Notes Monogr. Ser. 22), Institute of Mathematical Statistics, Hayward, CA, pp. 66-75.

[7] Hardy, G. H., Littlewood, J. E. and Pólya, G. (1929). Some simple inequalities satisfied by convex functions. Messenger Math. 58, 145-152. 
[8] Hardy, G. H., Littlewood, J. E. and Pólya, G. (1934). Inequalities. Cambridge University Press.

[9] Hardy, G. H., Littlewood, J. E. and Pólya, G. (1952). Inequalities, 2nd edn. Cambridge University Press.

[10] Huang, J. S. (1989). Moment problem of order statistics: a review. Internat. Statist. Rev. 57, 59-66.

[11] Johnson, N. L., Kotz, S. And Kemp, A. W. (1992). Univariate Discrete Distributions, 2nd edn. John Wiley, New York.

[12] Marshall, A. W. And Olkin, I. (1979). Inequalities: Theory of Majorization and Its Applications. Academic Press, Boston, MA.

[13] Müller, A. And Stoyan, D. (2002). Comparison Methods for Stochastic Models and Risks. John Wiley, New York.

[14] Rüschendorf, L. (1981). Ordering of distributions and rearrangement of functions. Ann. Prob. 9, $276-283$.

[15] Shaked, M. and Shanthikumar, J. G. (1994). Stochastic Orders and Their Applications. Academic Press, New York.

[16] Stoyan, D. (1983). Comparison Methods for Queues and Other Stochastic Models. John Wiley, New York.

[17] SzeKLI, R. (1987). A note on moment inequalities for order statistics from star-shaped distributions. Zastos. Mat. 19, 65-68.

[18] Szekli, R. (1995). Stochastic Ordering and Dependence in Applied Probability (Lecture Notes Statist. 97). Springer, New York.

[19] WANG, S. S. AND Young, V. R. (1998). Ordering risks: expected utility theory versus Yaari's dual theory of risk. Insurance Math. Econom. 22, 145-161. 